\title{
Triangle-free Subgraphs of Hypergraphs
}

\author{
Jiaxi Nie $^{*} \quad$ Sam Spiro ${ }^{\dagger} \quad$ Jacques Verstraëte ${ }^{\ddagger}$
}

May 11, 2020

\begin{abstract}
In this paper, we consider an analog of the well-studied extremal problem for triangle-free subgraphs of graphs for uniform hypergraphs. A loose triangle is a hypergraph $T$ consisting of three edges $e, f$ and $g$ such that $|e \cap f|=|f \cap g|=|g \cap e|=1$ and $e \cap f \cap g=\emptyset$. We prove that if $H$ is an $n$-vertex $r$-uniform hypergraph with maximum degree $\triangle$, then as $\triangle \rightarrow \infty$, the number of edges in a densest $T$-free subhypergraph of $H$ is at least

$$
\frac{e(H)}{\triangle^{\frac{r-2}{r-1}+o(1)}}
$$

For $r=3$, this is tight up to the $o(1)$ term in the exponent. We also show that if $H$ is a random $n$-vertex triple system with edge-probability $p$ such that $p n^{3} \rightarrow \infty$ as $n \rightarrow \infty$, then with high probability as $n \rightarrow \infty$, the number of edges in a densest $T$-free subhypergraph is

$$
\min \left\{(1-o(1)) p\left(\begin{array}{l}
n \\
3
\end{array}\right), p^{\frac{1}{3}} n^{2-o(1)}\right\} .
$$

We use the method of containers together with probabilistic methods and a connection to the extremal problem for arithmetic progressions of length three due to Ruzsa and Szemerédi.
\end{abstract}

\section{Introduction}

The Turán numbers for a graph $F$ are the quantities $\operatorname{ex}(n, F)$ denoting the maximum number of edges in an $F$-free $n$-vertex graph. The study of Turán numbers is a cornerstone of extremal graph theory, going back to Mantel's Theorem [22] and Turán's Theorem [29]. A more general problem involves studying ex $(G, F)$, which is the maximum number of edges in an $F$-free subgraph of a graph $G$. Some celebrated open problems are instances of this problem, such as the case when $G$ is the $n$-dimensional hypercube - see Conlon [7] for recent results.

In the case that $F$ is a triangle, $\operatorname{ex}(G, F) \geq \frac{1}{2} e(G)$ for every graph $G$, which can be seen by taking a maximum cut of $G$, which is essentially tight. In the case $G=G_{n, p}$, the Erdös-Rényi random

\footnotetext{
*Dept. of Mathematics, UCSD jin019@ucsd.edu

${ }^{\dagger}$ Dept. of Mathematics, UCSD sspiro@ucsd.edu. This material is based upon work supported by the National Science Foundation Graduate Research Fellowship under Grant No. DGE-1650112.

${ }^{\ddagger}$ Dept. of Mathematics, UCSD jverstra@math.ucsd.edu
} 
graph, $\operatorname{ex}(G, F) \sim \frac{1}{2} p\left(\begin{array}{c}n \\ 2\end{array}\right)$ with high probability provided $p$ is not too small, and furthermore every maximum triangle-free subgraph is bipartite - see di Marco and Kahn [10] and also Kohayakawa, Euczak and Rödl [21] and di Marco, Hamm and Kahn [9] for related stability results. The study of $F$-free subgraphs of random graphs when $F$ has chromatic number at least three is undertaken in seminal papers of Friedgut, Rödl and Schacht [16], Conlon and Gowers [8], and Schacht [28].

\subsection{Triangle-free subgraphs of hypergraphs}

In this paper, we consider a generalization of the problem of determining $\operatorname{ex}(G, F)$ when $F$ is a triangle to uniform hypergraphs. We write $r$-graph instead of $r$-uniform hypergraph. If $G$ and $F$ are $r$-graphs, then $\operatorname{ex}(G, F)$ denotes the maximum number of edges in an $F$-free subgraph of $G$. A loose triangle is a hypergraph $T$ consisting of three edges $e, f$ and $g$ such that $|e \cap f|=|f \cap g|=|g \cap e|=1$ and $e \cap f \cap g=\emptyset$. We write $T^{r}$ for the loose $r$-uniform triangle. The Turán problem for loose triangles in $r$-graphs was essentially solved by Frankl and Füredi [15], who showed for each $r \geq 3$ that $\operatorname{ex}\left(n, T^{r}\right)=\left(\begin{array}{c}n-1 \\ r-1\end{array}\right)$ for $n$ large enough, with equality only for the $r$-graph $S_{n}^{r}$ of all $r$-sets containing a single vertex. We remark that the Turán problem for $r$-graphs is notoriously difficult in general, and the asymptotic behavior of $\operatorname{ex}\left(n, K_{t}^{r}\right)$ is a well-known open problem of Erdös [11] - the celebrated Turán conjecture states $\operatorname{ex}\left(n, K_{4}^{3}\right) \sim \frac{5}{9}\left(\begin{array}{l}n \\ 3\end{array}\right)$.

The extremal problem for loose triangles is closely connected to the extremal problem for three-term arithmetic progressions in sets of integers. Specifically, Ruzsa and Szemerédi [26] made the following connection. If $\Gamma$ is an abelian group and $A \subseteq \Gamma$, define the tripartite linear triple system $H(A, \Gamma)$ whose parts are equal to $\Gamma$ and where $(\gamma, \gamma+a, \gamma+2 a)$ is an edge if $a \in A$. In other words, the edges are three-term progressions whose common difference is in $A$. One can then see that $H(A, \Gamma)$ has $|A||\Gamma|$ edges and is triangle-free whenever $A$ has no three term arithmetic progression. Ruzsa and Szemerédi [26] showed that every $n$-vertex triangle-free linear triple system has $o\left(n^{2}\right)$ edges, and applying this to $H(A, \Gamma)$ one obtains Roth's Theorem [24] that $|A|=o(|\Gamma|)$. A construction of Behrend [6] gives in $\mathbb{Z} / n \mathbb{Z}$ a set $A$ without three-term progressions of size $n / \exp (O(\sqrt{\log n}))$, and so $H(A, \mathbb{Z} / n \mathbb{Z})$ has $n^{2-o(1)}$ edges in this case. Erdős, Frankl, and Rödl [12] extended these ideas to $r$-uniform hypergraphs, giving the following.

Theorem 1.1 (Ruzsa and Szemerédi [26]; Erdős, Frankl, and Rödl [12]). For all $n$ there exists an $n$-vertex $r$-graph which is linear, loose triangle-free, and which has $n^{2-o(1)}$ edges as $n \rightarrow \infty$.

This theorem is an important ingredient for our first theorem, giving a general lower bound on the number of edges in a densest triangle-free subgraphs of $r$-graphs:

Theorem 1.2. Let $r \geq 3$ and let $G$ be an $r$-graph with maximum degree $\triangle$. Then as $\triangle \rightarrow \infty$,

$$
\operatorname{ex}\left(G, T^{r}\right) \geq \triangle^{-\frac{r-2}{r-1}-o(1)} e(G) .
$$

If a positive integer $t$ is chosen so that $\left(\begin{array}{c}t-1 \\ r-1\end{array}\right)<\Delta \leq\left(\begin{array}{c}t \\ r-1\end{array}\right)$ and $t \mid n$, then the $n$-vertex $r$-graph $G$ consisting of $n / t$ disjoint copies of a clique $K_{t}^{r}$ has maximum degree at most $\triangle$ whereas

$$
\operatorname{ex}\left(G, T^{r}\right)=\left(\begin{array}{l}
t-1 \\
r-1
\end{array}\right) \frac{n}{t}=\frac{r}{t} e(G)=O\left(\triangle^{-\frac{1}{r-1}}\right) \cdot e(G) .
$$


Here we used the result of Frankl and Füredi [15] that $S_{t}^{r}$ is the extremal $T^{r}$-free subgraph of $K_{t}^{r}$ for $t$ large enough. Therefore for $r=3$, Theorem 1.2 is sharp up to the $o(1)$ term in the exponent of $\triangle$. For $r \geq 4$, the best construction we have gives the following proposition:

Proposition 1.3. For $r \geq 4$ there exists an $r$-graph $G$ with maximum degree $\triangle$ such that as $\triangle \rightarrow \infty$,

$$
\operatorname{ex}\left(G, T^{r}\right)=O\left(\triangle^{-\frac{1}{2}}\right) \cdot e(G) .
$$

We leave it as an open problem to determine the smallest $c$ such that $\operatorname{ex}\left(G, T^{r}\right) \geq \triangle^{-c-o(1)} \cdot e(G)$ for every graph $G$ of maximum degree $\triangle$. We conjecture the following for $r=3$ :

Conjecture 1.4. For $\triangle \geq 1$, there exists a triple system $G$ with maximum degree $\triangle$ such that as $\triangle \rightarrow \infty$, every $T^{3}$-free subgraph of $G$ has $o\left(\triangle^{-1 / 2}\right) \cdot e(G)$ edges.

\subsection{Triangle-free subgraphs of random hypergraphs}

Our next set of results concern random hosts. To this end, we say that a statement depending on $n$ holds asymptotically almost surely (abbreviated a.a.s.) if the probability that it holds tends to 1 as $n$ tends to infinity. Let $G_{n, p}^{r}$ denote random $r$-graph where edges of $K_{n}^{r}$ are sampled independently with probability $p$. For the $r=2$ case we simply write $G_{n, p}$.

A central conjecture of Kohayakawa, Łuczak and Rödl [21] was resolved independently by Conlon and Gowers [8] and by Schacht [28], and determines the asymptotic value of $\operatorname{ex}\left(G_{n, p}, F\right)$ whenever $F$ has chromatic number at least three. The situation when $F$ is bipartite is more complicated, partly due to the fact that the order of magnitude of Turán numbers ex $(n, F)$ is not known in general - see Füredi and Simonovits [17] for a survey of bipartite Turán problems. The case of even cycles was studied by Kohayakawa, Kreuter and Steger [20] and Morris and Saxton [23] and complete bipartite graphs were studied by Morris and Saxton [23] and by Balogh and Samotij [5].

If $F$ consists of two disjoint $r$-sets, then $\operatorname{ex}(n, F)$ is given by the celebrated Erdös-Ko-Rado Theorem [13], and $\operatorname{ex}(n, F)=\left(\begin{array}{c}n-1 \\ r-1\end{array}\right)$. A number of researchers studied $\operatorname{ex}\left(G_{n, p}^{r}, F\right)$ in this case [2], with the main question being the smallest value of $p$ such that an extremal $F$-free subgraph of $G_{n, p}^{r}$ consists of all $r$-sets on a vertex of maximum degree - $(1+o(1)) p\left(\begin{array}{c}n-1 \\ r-1\end{array}\right)$ edges. The same subgraphs are also $T^{r}$-free, however the extremal subgraphs in that case are denser and appear to be more difficult to describe. Our second main result is as follows:

Theorem 1.5. For all $n \geq 2$ and $p=p(n) \leq 1$ with $p n^{3} \rightarrow \infty$ as $n \rightarrow \infty$, there exist a constant $c>0$ such that asymptotically almost surely

$$
\min \left\{(1-o(1)) p\left(\begin{array}{c}
n \\
3
\end{array}\right), p^{\frac{1}{3}} n^{2} e^{-c \sqrt{\log n}}\right\} \leq \operatorname{ex}\left(G_{n, p}^{3}, T^{3}\right) \leq \min \left\{(1+o(1)) p\left(\begin{array}{c}
n \\
3
\end{array}\right), p^{\frac{1}{3}} n^{2+o(1)}\right\},
$$

and more accurately, for any constant $\delta>0$, when $n^{-3 / 2+\delta} \leq p \leq n^{-\delta}$, we have

$$
\operatorname{ex}\left(G_{n, p}^{3}, T^{3}\right) \leq p^{\frac{1}{3}} n^{2}(\log n)^{c} .
$$


We believe that perhaps the lower bound is closer to the truth.

Since $G_{n, p}$ for $p>n^{-2+o(1)}$ has maximum degree $\triangle \sim p\left(\begin{array}{c}n-1 \\ 2\end{array}\right)$ asymptotically almost surely, Theorem 1.2 only gives $\operatorname{ex}\left(G_{n, p}^{3}, T^{3}\right) \geq p^{1 / 2-o(1)} n^{2}$ a.a.s. The upper bound in Theorem 1.5 employs the method of containers developed by Balogh, Morris and Samotij [3] and Saxton and Thomason [27].

We do not have tight bounds for $\operatorname{ex}\left(G_{n, p}^{r}, T^{r}\right)$ in general for all $p$ and $r \geq 4$. Partial results and conjectures are discussed in the concluding remarks.

\subsection{Counting triangle-free hypergraphs}

Balogh, Narayanan and Samotij [4] showed that the number of triangle-free $n$-vertex $r$-graphs is $2^{\Theta\left(n^{r-1}\right)}$ using the method of containers. Note that a lower bound follows easily by counting all subgraphs of the $r$-graph $S_{n}^{r}$ on $n$ vertices consisting of all $r$-sets containing a fixed vertex. In this section, we adapt the methods to counting triangle-free hypergraphs with a specified number of edges.

Theorem 1.6. Let $N_{3}(n, m)$ denote the number of $T^{3}$-free 3-graphs with $n$ vertices and $m$ edges. Let $\epsilon(n)$ be a function such that $\frac{\epsilon(n) \log n}{\log \log n} \rightarrow \infty$ as $n \rightarrow \infty$. Let $\delta=\delta(n)$ be a function such that $\epsilon(n)<\delta<1 / 2-\epsilon(n)$ and let $m=n^{2-\delta}$. Then

$$
N_{3}(n, m) \leq\left(\frac{n^{2}}{m}\right)^{3 m+o(m)} .
$$

We note that an analog of Theorem 1.6 for graphs was proven by Balogh and Samotij [5]. The upper bound on ex $\left(G_{n, p}^{3}, T^{3}\right)$ in Theorem 1.5 will follow quickly from the bound on $N_{3}(n, m)$ in Theorem 1.6 by taking $m=p^{1 / 3-o(1)} n^{2}$.

\section{Proofs of Theorem 1.2 and Proposition 1.3}

For graphs, Foucaud, Krivelevich and Perarnau [14] used certain random homomorphisms to obtain good lower bounds on $\operatorname{ex}(G, F)$. We briefly summarize these ideas. Let $\mathcal{M}(F)$ denote the family of graphs $F^{\prime}$ with $e\left(F^{\prime}\right)=e(F)$ and which can be obtained from $F$ by identifying vertices. Let $H$ be an $\mathcal{M}(F)$-free graph with many edges, which we will use as a template for our subgraph of $G$. Specifically, we take a random mapping $\chi: V(G) \rightarrow V(H)$ and then constructs a subgraph $G^{\prime} \subseteq G$ such that $u v \in E\left(G^{\prime}\right)$ if and only if $\chi(u) \chi(v) \in E(H)$ and such that $\chi(u) \chi(v) \neq \chi(u) \chi(w)$ for any other edge $u w \in E(G)$ (that is, we do not keep edges which are incident and map to the same vertex). It turns out that $G^{\prime}$ will be $F$-free because $H$ is $\mathcal{M}(F)$-free, and in expectation $G^{\prime}$ will have many edges provided $H$ does.

For general $r$-graphs, it is not immediately clear how to extend these ideas in such a way that we can both construct a subgraph with many edges and such that the subgraph is $F$-free. Fortunately for $T^{r}$ we are able to do this. In particular, for this case it turns out we can ignore the family $\mathcal{M}\left(T^{r}\right)$ provided our template $r$-graph is linear. This is where the Ruzsa-Szemeredi construction of Theorem 1.1 plays its crucial role. 
Proof of Theorem 1.2. Let $t$ be an integer to be determined later. Let $\chi$ be a random map from $V(G)$ to $[t]$ and $G_{t}$ the $r$-graph from Theorem 1.1. For ease of notation define $\chi(e)=\left\{\chi\left(v_{1}\right), \ldots, \chi\left(v_{r}\right)\right\}$ when $e=\left\{v_{1}, \ldots, v_{r}\right\}$. Let $G^{\prime}$ be the subgraph of $G$ which contains the edge $e$ if and only if

(1) $\chi(e)$ is an edge of $G_{t}$, and

(2) $\chi\left(e^{\prime}\right) \not \subset \chi(e)$ for any $e^{\prime} \in E(G)$ with $\left|e \cap e^{\prime}\right|=1$.

We claim that $G^{\prime}$ is $T^{r}$-free. Indeed, let $T$ be a $T^{r}$ of $G^{\prime}$, say with edges $e_{1}, e_{2}, e_{3}$ and $e_{i} \cap e_{j}=\left\{x_{i j}\right\}$ for $i \neq j$. Because $G_{t}$ is linear, if $e, e^{\prime}$ are (possibly non-distinct) edges of $G_{t}$, then $\left|e \cap e^{\prime}\right|$ is either 0,1 , or 3 . Note that $\chi\left(e_{i}\right), \chi\left(e_{j}\right)$ are edges of $G_{t}$ by (1). Because $e_{i} \cap e_{j}=\left\{x_{i j}\right\}$ for $i \neq j, \chi\left(x_{i j}\right) \in \chi\left(e_{i}\right) \cap \chi\left(e_{j}\right)$, and by (2) the size of this intersection is strictly less than $r$. Thus $\chi\left(e_{i}\right) \cap \chi\left(e_{j}\right)=\left\{\chi\left(x_{i j}\right)\right\}$. Further, we must have, say, $\chi\left(x_{i j}\right) \neq \chi\left(x_{i k}\right)$ for $k \neq i, j$. This is because (1) guarantees that $\chi(x)$ is a distinct element for each $x \in e_{i}$, so in particular this holds for $x_{i j}, x_{i k} \in e_{i}$. In total this implies $\chi\left(e_{1}\right), \chi\left(e_{2}\right), \chi\left(e_{3}\right)$ forms a $T^{r}$ in $G_{t}$, a contradiction.

We wish to compute how large $e\left(G^{\prime}\right)$ is in expectation. Fix some $e \in E(G)$. The probability that $e$ satisfies (1) is exactly $e\left(G_{t}\right) r ! / t^{r}$. Let $\left\{e_{1}, \ldots, e_{d}\right\}$ be the edges in $E(G)$ with $\left|e_{i} \cap e\right|=1$. Given that $e$ satisfies $(1)$, the probability that $\chi\left(e_{1}\right) \not \subset \chi(e)$ is exactly $1-(r / t)^{r-1}$. Note that for any $v \notin e \cup e_{1}$, the event $\chi(v) \in \chi(e)$ is independent of the event $\chi\left(e_{1}\right) \not \subset \chi(e)$, so we have

$$
\operatorname{Pr}\left[\chi(v) \in \chi(e) \mid e \text { satisfies }(1), \chi\left(e_{1}\right) \not \subset \chi(e)\right]=\frac{r}{t} .
$$

On the other hand, if $v \in e_{1} \backslash e$, then

$$
\operatorname{Pr}\left[\chi(v) \in \chi(e) \mid e \text { satisfies }(1), \chi\left(e_{1}\right) \not \subset \chi(e)\right]<\frac{r}{t},
$$

as knowing some subset containing $\chi(v)$ is not contained in $\chi(e)$ makes it less likely that $\chi(v) \in \chi(e)$. By applying these observations to each vertex of $e_{2} \backslash e$, we conclude that

$$
\operatorname{Pr}\left[\chi\left(e_{2}\right) \not \subset \chi(e) \mid e \text { satisfies }(1), \chi\left(e_{1}\right) \not \subset \chi(e)\right] \geq 1-\left(\frac{r}{t}\right)^{r-1} .
$$

By repeating this logic for each $e_{i}$, and using that $e\left(G_{t}\right)=t^{2-o(1)}$, we conclude that

$$
\operatorname{Pr}[e \text { satisfies (1), (2) }] \geq \frac{e\left(G_{t}\right) r !}{t^{r}}\left(1-\left(\frac{r}{t}\right)^{r-1}\right)^{r \Delta}=t^{2-r-o(1)}\left(1-\left(\frac{r}{t}\right)^{r-1}\right)^{r \Delta} .
$$

By taking $t=r(r \triangle)^{1 /(r-1)}$ and using that $\left(1-x^{-1}\right)^{x}$ is a decreasing function in $x$, we conclude by linearity of expectation that

$$
\mathbb{E}\left[e\left(G^{\prime}\right)\right] \geq \triangle^{-1+\frac{1}{r-1}-o(1)} \cdot e(G)
$$

In particular, there exists some $T^{r}$-free subgraph of $G$ with at least this many edges, giving the desired result.

We close this section with a proof of Proposition 1.3. 
Proof of Proposition 1.3. By Rödl [25], there exists an $r$-graph $G$ with $\Theta\left(n^{3}\right)$ edges such that every three vertices is contained in at most one edge. Let $G^{\prime}$ be a $T^{r}$-free subgraph of $G$. Define $G^{\prime \prime}$ by deleting every edge of $G^{\prime}$ which contains two vertices that are contained in at most $2 r$ edges. Note that $e\left(G^{\prime}\right)-e\left(G^{\prime \prime}\right) \leq 2 r\left(\begin{array}{l}n \\ 2\end{array}\right)$.

Assume $G^{\prime \prime}$ contains an edge $e=\left\{v_{1}, \ldots, v_{r}\right\}$. Because $v_{1}, v_{2}$ are contained in an edge of $G^{\prime \prime}$, there exist a set $E_{12} \subseteq E\left(G^{\prime}\right)$ of at least $2 r+1$ many edges containing $v_{1}$ and $v_{2}$. As $G$ contained at most one edge containing $v_{1}, v_{2}$, and $v_{3}$, any $e_{12} \neq e$ in $E$ does not contain $v_{3}$. Because $v_{2}, v_{3}$ are contained in an edge of $G^{\prime \prime}$, there exists a set $E_{23} \subseteq E\left(G^{\prime}\right)$ of at least $2 r+1 \geq r+1$ edges containing $v_{2}, v_{3}$. Because $G$ contains at most one edge containing $v_{2}, v_{3}, u_{i}$ for any $u_{i} \in e_{12}$, we conclude that there exists some $e_{23} \in E_{23}$ such that $e_{12} \cap e_{23}=\left\{v_{2}\right\}$. Similarly we can find some $e_{13} \in E\left(G^{\prime}\right)$ such that $v_{1}, v_{3} \in e_{13}$ and such that $e_{13} \cap e_{12}=\left\{v_{1}\right\}, e_{13} \cap e_{23}=\left\{v_{3}\right\}$. These three edges form a $T^{r}$ in $G^{\prime}$, a contradiction. We conclude that $G^{\prime \prime}$ contains no edges, and hence $e\left(G^{\prime}\right) \leq 2 r\left(\begin{array}{c}n \\ 2\end{array}\right)$ for any $T^{r}$-free subgraph $G^{\prime}$ of $G$. As $G$ has maximum degree $\triangle=\Theta\left(n^{2}\right)$, we conclude that $\operatorname{ex}\left(G, T^{r}\right)=O\left(n^{2}\right)=O\left(\triangle^{-1 / 2}\right) \cdot e(G)$.

We note that one can replace the $G$ used in the above proof with an appropriate Steiner system to obtain a regular graph which serves as an upper bound. It has recently been proven by Keevash [19] and Glock, Kühn, Lo, and Osthus [18] that such Steiner systems exist whenever $n$ satisfies certain divisibility conditions and is sufficiently large.

\section{Proof of Theorem 1.5: Lower Bound.}

As noted in the introduction, the bound of Theorem 1.2 is sharp for $r=3$ by considering the disjoint union of cliques, so we can not improve upon this bound in general. However, we are able to do better when $G$ contains few copies of $T^{r}$ by using a deletion argument.

Proposition 3.1. Let $R(G)$ denote the number of copies of $T^{r}$ in the r-graph $G$. Then for any integer $t \geq 1$,

$$
\operatorname{ex}\left(G, T^{r}\right) \geq\left(e(G) t^{2-r}-R(G) t^{5-3 r}\right) e^{-c \sqrt{\log t}}
$$

Proof. Let $\chi$ be a random map from $V(G)$ to $[t]$ and $G_{t}$ the $r$-graph from Theorem 1.1. For ease of notation, if $e=\left\{v_{1}, \ldots, v_{r}\right\}$ we define $\chi(e):=\left\{\chi\left(v_{1}\right), \ldots, \chi\left(v_{r}\right)\right\}$. Let $G^{\prime}$ be the subgraph of $G$ which contains the edge $e$ if and only if $\chi(e)$ is an edge of $G_{t}$.

We claim that $e_{1}, e_{2}, e_{3} \in E\left(G^{\prime}\right)$ form a $T^{r}$ in $G^{\prime}$ if and only if $e_{1}, e_{2}, e_{3}$ form a $T^{r}$ in $G$ and $\chi\left(e_{1}\right)=\chi\left(e_{2}\right)=\chi\left(e_{3}\right)$ is an edge of $G_{t}$. Indeed, the backwards direction is clear. Assume for contradiction that these edges form a $T^{r}$ in $G^{\prime}$ and that $e_{1} \neq e_{2}$. Let $x_{i j}$ for $i \neq j$ be such that $e_{i} \cap e_{j}=\left\{x_{i j}\right\}$. Because $G_{t}$ is linear, if $e, e^{\prime}$ are (possibly non-distinct) edges of $G_{t}$, then $\left|e \cap e^{\prime}\right|$ is either 0,1 , or $r$. Because each $e_{i}$ is in $E\left(G^{\prime}\right)$, we have $\chi\left(e_{i}\right) \in E\left(G_{t}\right)$ by construction. In particular, as $e_{1} \cap e_{2}=\left\{x_{12}\right\}$ and $\chi\left(e_{1}\right) \neq \chi\left(e_{2}\right)$, we must have $\chi\left(e_{1}\right) \cap \chi\left(e_{2}\right)=\left\{\chi\left(x_{12}\right)\right\}$. As $e_{3}$ contains elements in and not in $e_{1}$ (namely $x_{13}$ and $x_{23}$ ), we must have $\chi\left(e_{1}\right) \cap \chi\left(e_{3}\right)=\left\{\chi\left(x_{13}\right)\right\}$. Similarly we have $\chi\left(e_{2}\right) \cap \chi\left(e_{3}\right)=\left\{\chi\left(x_{23}\right)\right\}$. Because $\chi\left(e_{i}\right)$ is an $r$-set for each $i$, we have $\chi\left(x_{i j}\right) \neq \chi\left(x_{i k}\right)$ for $\{i, j, k\}=\{1,2,3\}$. Thus $\chi\left(e_{1}\right), \chi\left(e_{2}\right), \chi\left(e_{3}\right)$ form a $T^{r}$ in $G_{t}$, a contradiction. 
Let $G^{\prime \prime} \subseteq G^{\prime}$ be a subgraph obtained by deleting an edge from each $T^{r}$ of $G^{\prime}$. By construction $G^{\prime \prime}$ is $T^{r}$-free. We conclude by linearity of expectation that

$$
\begin{aligned}
\operatorname{ex}\left(G, T^{r}\right) & \geq \mathbb{E}\left[e\left(G^{\prime \prime}\right)\right] \geq \mathbb{E}\left[e\left(G^{\prime}\right)-R\left(G^{\prime}\right)\right] \\
& =\frac{e\left(G_{t}\right) r !}{t^{r}} e(G)-\frac{e\left(G_{t}\right) r !}{t^{3 r-3}} R(G) \\
& \geq\left(e(G) t^{2-r}-R(G) t^{5-3 r}\right) e^{-c \sqrt{\log t}}
\end{aligned}
$$

Corollary 3.2. For any integer $r \geq 3$, and function $p=p(n) \leq 1$ such that $p^{2 /(2 r-3)} n \geq 2$, we have

$$
\mathbb{E}\left[\operatorname{ex}\left(G_{n, p}^{r}, T^{r}\right)\right] \geq p^{\frac{1}{2 r-3}} n^{2} e^{-c \sqrt{n}}
$$

for some constant $c>0$.

Proof. Note for $n \geq 4$ that $\mathbb{E}\left[e\left(G_{n, p}^{r}\right)\right]=p\left(\begin{array}{l}n \\ r\end{array}\right) \geq p n^{r} /(2 r)^{r} \geq p n^{r} / r^{2 r}$, and that $\mathbb{E}\left[R\left(G_{n, p}^{r}\right)\right] \leq p^{3} n^{3 r-3}$. Plugging these into the bound of Proposition 3.1 gives

$$
\mathbb{E}\left[\operatorname{ex}\left(G_{n, p}^{r}, T^{r}\right)\right] \geq\left(p n^{r} t^{2-r}-p^{3} n^{3 r-3} t^{5-3 r}\right) e^{-c \sqrt{\log t}} .
$$

Take $t=p^{2 /(2 r-3)} n^{1 / 2}$, we conclude for sufficiently large $n$ that

$$
\mathbb{E}\left[\operatorname{ex}\left(G_{n, p}^{r}, T^{r}\right)\right] \geq p^{\frac{1}{2 r-3}} n^{2} e^{-c \sqrt{\log n}} .
$$

To get the a.a.s. result of Theorem 1.5, we use Azuma's inequality (See for example in Alon and Spencer [1]) applied to the edge exposure martingale.

Lemma 3.3. Let $f$ be a function on $r$-graphs such that $|f(G)-f(H)| \leq 1$ whenever $H$ is $G$ with exactly one edge added or deleted. Then for any $\lambda>0$,

$$
\operatorname{Pr}\left[\left|f\left(G_{n, p}^{r}\right)-\mathbb{E}\left[f\left(G_{n, p}^{r}\right)\right]\right|>\lambda \sqrt{\left(\begin{array}{l}
n \\
r
\end{array}\right)}\right]<e^{-\frac{\lambda^{2}}{2}} .
$$

Proof of Theorem 1.5: Lower Bounds. Let $\epsilon(n)=e^{k \sqrt{\log n}}$, where $k>0$ is some large enough constant. For $p \leq n^{-3 / 2} / \epsilon(n)$, it is not difficult to show that a.a.s. $G_{n, p}^{3}$ contains $o\left(p n^{3}\right)$ copies of $T^{3}$, and by deleting an edge from each of these loose cycles we see that $\operatorname{ex}\left(G_{n, p}^{3}, T^{3}\right)=\left(1-o(1) p\left(\begin{array}{l}n \\ 3\end{array}\right)\right.$ a.a.s.

For $n^{-3 / 2} / \epsilon(n) \leq p \leq n^{-3 / 2} \epsilon(n)$, we do an extra round of random sampling on the edges of $G_{n, p}^{r}$ and keep each edge with probability $p^{\prime}:=\epsilon(n)^{-2}$. The $r$-graph we obtained is equivalent to $G_{n, p p^{\prime}}^{r}$, with $p p^{\prime} \leq n^{-3 / 2} / \epsilon(n)$. Thus $\left.\operatorname{ex}\left(G_{n, p}^{3}, T^{3}\right)=(1-o(1)) p p^{\prime}\left(\begin{array}{l}n \\ 3\end{array}\right)=(1-o(1)) p\left(\begin{array}{l}n \\ 3\end{array}\right) / \epsilon(n)^{2}\right)$ a.a.s. Using $p \geq n^{-3 / 2} / \epsilon(n)$, we conclude that $\operatorname{ex}\left(G_{n, p}^{3}, T^{3}\right) \geq p^{1 / 3} n^{2} e^{-c \sqrt{\log n}}$ a.a.s. in this range.

We now consider $p \geq n^{-3 / 2} \epsilon(n)$. The bound in expectation follows from Corollary 3.2. To show that this result holds a.a.s., we observe that $f(G)=\operatorname{ex}\left(G, T^{3}\right)$ satisfies the conditions of Lemma 3.3. 
For ease of notation let $X_{n, p}=\operatorname{ex}\left(G_{n, p}^{3}, T^{3}\right)$ and let $B_{n, p}=p^{1 / 3} n^{2} e^{-c \sqrt{\log n}}$ be the lower bound for $\mathbb{E}\left[X_{n, p}\right]$ given in Corollary 3.2. Setting $\lambda=\frac{1}{2} B_{n, p}\left(\begin{array}{l}n \\ 3\end{array}\right)^{-1 / 2}$ and applying Azuma's inequality, we find

$$
\begin{aligned}
\operatorname{Pr}\left[X_{n, p}<\frac{1}{2} B_{n, p}\right] & \leq \operatorname{Pr}\left[X_{n, p}-\mathbb{E}\left[X_{n, p}\right]<\lambda\left(\begin{array}{l}
n \\
3
\end{array}\right)^{\frac{1}{2}}\right] \\
& \leq \operatorname{Pr}\left[\left|X_{n, p}-\mathbb{E}\left[X_{n, p}\right]\right|<\lambda\left(\begin{array}{l}
n \\
3
\end{array}\right)^{\frac{1}{2}}\right] \leq \exp \left(-\frac{\lambda^{2}}{2}\right) .
\end{aligned}
$$

Note that for $p \geq n^{-3 / 2} \epsilon(n)$ we have $\lambda \geq e^{(k / 3-c) \sqrt{\log n}} \rightarrow \infty$ as $n \rightarrow \infty$. So we conclude the a.a.s. result.

\section{Containers}

The method of containers developed by Balogh, Morris and Samotij [3] and Saxton and Thomason [27] is a powerful technique that has been used to solve a number of combinatorial problems. Roughly, the idea is for a suitable hypergraph $H$ to find a family of sets $\mathcal{C}$ which contain every independent set of $H$, and in such a way that $|\mathcal{C}|$ is small and each $C \in \mathcal{C}$ contains few edges. For example, by letting $H$ be the 3-uniform hypergraph where each edge is a $K_{3}$ in some graph $G$, we see that independent sets of $H$ correspond to triangle-free subgraphs of $G$. The existence of containers then allows us to better understand how these subgraphs of $G$ behave.

We proceed with the technical details of this approach. Given an $r$-graph $H=(V, E)$, let $v(H)=|V|$, $e(H)=|E|$, and let $\mathcal{P}(V)$ be the family of subsets of $V$. For $A$ a set of vertices in $H$, let $d(A)$ be the number of edges in $H$ that contain $A$. Let $\bar{d}(H)$ be the average degree of $H$, and let $\triangle_{j}(H)=$ $\max _{|A|=j} d(A)$. In order to establish our upper bounds, we need to use the following container lemma for hypergraphs:

Lemma 4.1 (Balogh, Morris and Samotij [3]). Let $r, b, l \in \mathbb{N}, \delta=2^{-r(r+1)}$, and $H=(V, E)$ an r-graph such that

$$
\triangle_{j}(H) \leq\left(\frac{b}{v(H)}\right)^{j-1} \frac{e(H)}{l}, j \in\{1,2, \ldots, r\} .
$$

Then there exists a collection $\mathcal{C}$ of subsets of $V$ and a function $f: \mathcal{P}(V) \rightarrow \mathcal{C}$ such that:

(1) For every independent set $I$ of $H$, there exists $S \subset I$ with $|S| \leq(k-1) b$ such that $I \subset f(S)$.

(2) For every $C \in \mathcal{C},|C| \leq v(H)-\delta l$.

We will use this container lemma to give an upper bound for $N_{3}(n, m)$, which we recall is the number of $T^{3}$-free 3 -graphs with $n$ vertices and $m$ edges. The idea is to consider the 3 -graph $H$ with $V(H)=E\left(K_{n}^{r}\right)$ and $E(H)$ consisting of $T^{r}$ in $K_{n}^{r}$. Notice that the container lemma requires upper bounds for the maximum codegrees of the hypergraph. In order to meet this requirement, we will use a balanced-supersaturation lemma for $T^{r}$. 
Lemma 4.2 (Balogh, Narayanan and Skokan [4]). For every $r \geq 3$, there exists $c=c(r)$ such that the following holds for all $n$. Given any $r$-graph $G$ on $[n]$ with $e(G)=t n^{r-1}, t \geq 6(r-1)$, let $S=1$ if $r=3$ and $S=t n^{r-4}$ if $r \geq 4$. Then there exists a 3-graph $H$ on $E(G)$, where each edge of $H$ is a copy of $T^{r}$ in $G$, such that:

(1) $\bar{d}(H) \geq c^{-1} t^{3} S^{2}$.

(2) $\triangle_{j}(H) \leq c t^{5-2 j} S^{3-j}$,for $j=1,2$.

Using the previous two lemmas, we derive the following container lemma for $T^{3}$-free hypergraphs. Similar result for $T^{r}$-free hypergraphs can also be obtained using the same idea, and we briefly comment on these results in the concluding remarks.

Lemma 4.3. For any integer $n$ and positive number $t$ with $12 \leq t \leq\left(\begin{array}{l}n \\ 3\end{array}\right) / n^{2}$, there exists a collection $\mathcal{C}$ of subgraphs of $K_{n}^{3}$ such that for some constant $c$ :

(1) For any $T^{3}$-free subgraph $J$ of $K_{n}^{3}$, there exists $C \in \mathcal{C}$ such that $J \subset C$.

(2) $|\mathcal{C}| \leq \exp \left(\frac{c \log (t) n^{2}}{\sqrt{t}}\right)$

(3) For every $C \in \mathcal{C}, e(C) \leq t n^{2}$.

Proof. By Lemma 4.2, there exists a positive constant $c_{1}$ such that for any 3-graph $G$ on $[n]$ with $e(G)=t_{0} n^{2}$ and $t_{0} \geq t$, there exists a 3 -graph $H$ on $E(G)$ such that:

(1) Every edge of $H$ is a copy of $T^{3}$.

(2) $\bar{d}(H) \geq c_{1}^{-1} t_{0}^{3}$.

(3) $\triangle_{j}(H) \leq c_{1} t_{0}^{5-2 j}, j=1,2 . \triangle_{3}(H)=1$.

We can then use Lemma 4.1 on $H$ with $l=t_{0} n^{2} /\left(3 c_{1}^{2}\right)$ and $b=n^{2} / \sqrt{c_{1} t_{0}}$ to get a collection $\mathcal{C}$ of subgraphs of $G$ such that they contain all $T^{3}$-free subgraphs of $G$, and for each $C \in \mathcal{C}, e(C) \leq$ $(1-\epsilon) t_{0} n^{2}$ for some constant $\epsilon>0$. Also, we have

$$
|\mathcal{C}| \leq \sum_{s=1}^{2 b}\left(\begin{array}{c}
t n^{2} \\
s
\end{array}\right) \leq \exp \left(\frac{c_{2} \log \left(t_{0}\right) n^{2}}{\sqrt{t_{0}}}\right)
$$

for some constant $c_{2}>0$.

We use the above argument on $G=K_{n}^{3}$ to get a family of containers $\mathcal{C}_{1}$. Notice that the containers of $\mathcal{C}_{1}$ are also 3 -graphs on $[n]$, so we can repeat this argument on each $C \in \mathcal{C}_{1}$ with more than $t n^{2}$ edges to get a new collection of containers $\mathcal{C}_{2}$. We do this repeatedly until all containers have less than $t n^{2}$ edges. Since in each step the number of edges will decrease by a constant $(1-\epsilon)$, this process must stop after at most $\log (n / t) / \epsilon$ steps. For $k \geq 0$, define $t_{k+1}=(1-\epsilon) t_{k}$. Let $M$ be the 
largest integer such that $t_{M}>t$. Because $t_{0} \leq\left(\begin{array}{l}n \\ 3\end{array}\right) / n^{2}$, the number of containers we have in the end is less than

$$
\begin{aligned}
\prod_{i=0}^{M} \exp \left(\frac{c_{2} \log \left(t_{i}\right) n^{2}}{\sqrt{t_{i}}}\right) & =\exp \left(\sum_{i=0}^{M} \frac{c_{2} \log \left(t_{i}\right) n^{2}}{\sqrt{t_{i}}}\right) \\
& \leq \exp \left(\frac{c \log (t) n^{2}}{\sqrt{t}}\right)
\end{aligned}
$$

for some constant $c>0$.

With the lemma above, we are ready to bound $N_{3}(n, m)$.

Proof of Theorem 1.6. Let $\mathcal{C}$ be a collection of containers and $c$ a constant as in Lemma 4.3 with $t=n^{2 \delta+\epsilon_{1}(n)}$, where $\epsilon_{1}(n)=\frac{2 \log \log n}{\log n}$. By considering all subgraphs of each $C \in \mathcal{C}$ with $m=n^{2-\delta}$ edges, and recalling that $\epsilon(n)<\delta<1 / 2-\epsilon(n)$ and $\left(\begin{array}{l}n \\ k\end{array}\right) \leq(e n / k)^{k}$, we conclude that for some suitable $\epsilon(n)$,

$$
\begin{aligned}
N_{3}(n, m) & \leq \exp \left(\frac{c \log (t) n^{2}}{\sqrt{t}}\right) \cdot\left(\begin{array}{c}
t n^{2} \\
m
\end{array}\right) \\
& \leq \exp \left(c \log t \cdot \frac{m}{\log n}+\left(1+\left(3 \delta+\epsilon_{1}(n)\right) \log n\right) m\right) \\
& \leq \exp \left(\delta \log n \cdot m\left(3+(2+o(1)) \frac{\log \log n}{\delta \log n}\right)\right) \\
& \leq\left(\frac{n^{2}}{m}\right)^{3 m+o(m)} .
\end{aligned}
$$

We are now ready to prove the upper bound of Theorem 1.5

Proof of Theorem 1.5: Upper Bound. We will only present the proof of the upper in terms of o(1) for the whole range. The proof of the more accurate upper bound in the smaller range is essentially the same, with more careful and explicit computation for the $o(1)$ factor. For $p \leq n^{-3 / 2+o(1)}$, the proof for the upper bound is exactly the same as that for the lower bound. We now consider $n^{-3 / 2+\epsilon(n)} \leq p \leq n^{-\epsilon(n)}$ for some small function $\epsilon(n)=o(1)$. Our goal is to show

$$
\operatorname{Pr}\left[\operatorname{ex}\left(G_{n, p}^{3}, T^{3}\right) \geq m\right] \rightarrow 0, \text { as } n \rightarrow \infty,
$$

for some $m=p^{1 / 3} n^{2+o(1)}$. Let $X_{m}$ be the expected number of $T^{3}$-free subgraphs in $G_{n, p}^{3}$ with $m$ edges. By Theorem 1.6, when $n^{3 / 2+\epsilon_{1}(n)} \leq m \leq n^{2-\epsilon_{1}(n)}$ for some function $\epsilon_{1}(n)=o(1)$, there exist a function $\epsilon_{2}(n)=o(1)$ such that the expectation of $X_{m}$ satisfies

$$
\begin{aligned}
\mathbb{E}\left[X_{m}\right] & =N_{3}(n, m) \cdot p^{m} \\
& \leq\left(\frac{n^{2}}{m}\right)^{m\left(3+\epsilon_{2}(n)\right)} p^{m} \\
& =\left(\left(\frac{n^{2}}{m}\right)^{\left(3+\epsilon_{2}(n)\right)} p\right)^{m} .
\end{aligned}
$$


We can let $m=p^{1 / 3-\epsilon_{3}(n)} n^{2}$ for some small function $\epsilon_{3}(n)=o(1)$ such that

$$
\left(\frac{n^{2}}{m}\right)^{\left(3+\epsilon_{2}(n)\right)} p<1 .
$$

Also we can pick some suitable $\epsilon(n)$, so that $n^{3 / 2+\epsilon_{1}(n)} \leq m \leq n^{2-\epsilon_{1}(n)}$. Thus we have $\mathbb{E}\left[X_{m}\right] \rightarrow 0$ as $n \rightarrow \infty$. Then by Markov's inequality, we have

$$
\operatorname{Pr}\left[e x\left(G_{n, p}^{3}, T^{3}\right) \geq m\right]=\operatorname{Pr}\left[X_{m} \geq 1\right] \leq \mathbb{E}\left[X_{m}\right] \rightarrow 0 \text {, as } n \rightarrow \infty .
$$

So a.a.s. we have

$$
\operatorname{ex}\left(G_{n, p}^{3}, T^{3}\right)<m=p^{\frac{1}{3}} n^{2+o(1)} .
$$

Finally for $p \geq n^{-o(1)}$, we have $\operatorname{ex}\left(G_{n, p}^{3}, T\right)<\operatorname{ex}\left(K_{n}^{3}, T\right)=\Theta\left(n^{2}\right)=p^{1 / 3} n^{2+o(1)}$ a.a.s.

\section{Concluding Remarks}

- We are able to generalize Theorem 1.6 to $r$-graphs as follows:

Theorem 5.1. Let $N_{r}(n, m)$ denote the number of $T^{r}$-free $r$-graphs with $n$ vertices and $m$ edges. Let $r \geq 4,0<\delta<3 / 2$, and $m=n^{3-\delta}$. Then

$$
N_{r}(n, m) \leq\left(\frac{n^{r-1}}{m}\right)^{\left(1+\frac{2 \delta}{3 r-12+3 \delta}\right) m+o(m)} .
$$

When $r>4$, let $m=n^{3+\delta}$ with $\delta$ some constant satisfying $0<\delta<r-4$. Then we have

$$
N_{r}(n, m) \leq\left(\frac{n^{r-1}}{m}\right)^{m+o(m)} .
$$

This bound will also leads to an upper bound for $\operatorname{ex}\left(G_{n, p}^{r}, T^{r}\right)$ when $n^{-r+3 / 2+o(1)} \leq p \leq 1$, which is essentially tight for $p=p(n)$ with $n^{-r+4+o(1)} \leq p \leq 1$. However, there is a gap between the lower bound and upper bound in the range $n^{-r+3 / 2+o(1)} \leq p \leq n^{-r+4+o(1)}$.

- Using the same techniques for the $r=3$ case, we are able to show the following.

Theorem 5.2. For $r \geq 4$ and $0 \leq x \leq r$ a constant, let $p=n^{-r+x}$ and define

$$
f_{r}(x)=\lim _{n \rightarrow \infty} \log _{n} \mathbb{E}\left[\operatorname{ex}\left(G_{n, p}^{r}, T^{r}\right)\right] .
$$

Then for $0 \leq x \leq 3 / 2, f_{r}(x)=x ;$ for $4<x \leq r, f_{r}(x)=x-1$; and for $3 / 2<x \leq 4$, we have

$$
\max \left\{\frac{x+3 r-6}{2 r-3}, x-1\right\} \leq f_{r}(x) \leq \frac{3 x+3}{5} .
$$

The bounds for $x \leq 3 / 2$ come from deleting an edge from each triangle in $G_{n, p}^{r}$. For $x>3 / 2$, the upper bound follow from Theorem 5.1, the first lower bound follows from Corollary 3.2, and the second lower bound follows from taking every edge containing a given vertex. 
- We believe that the upper bound is perhaps closer to the truth and have the following conjecture.

Conjecture 5.3. For $r \geq 4$ and $0 \leq x \leq r$ a constant, let $p=n^{-r+x}$ and $f_{r}(x)$ as defined in Theorem 5.2. Then for $\frac{3}{2}<x \leq 4$,

$$
f_{r}(x)=\frac{3 x+3}{5}
$$

- For the deterministic case, we note that one can extend the proof of Theorem 1.2 to other $F$ by defining maps $\chi: V(G) \rightarrow V(H)$ for suitable $H$. In this case a second step must be done to effectively bound $\operatorname{ex}(G, F)$. We plan to do this in a followup paper.

\section{References}

[1] N. Alon and J. H. Spencer, The probabilistic method. John Wiley \& Sons, (2016).

[2] J. Balogh, T. Bohman and D. Mubayi, Erds-Ko-Rado in Random Hypergraphs. Combinatorics, Probability and Computing, 18(5) (2009), 629-646.

[3] J. Balogh, R. Morris, and W. Samotij, The method of hypergraph containers. Proceedings of the International Congress of Mathematicians-Rio de Janeiro. Vol. 3 (2018), 3045-3078

[4] J. Balogh, B. Narayanan and J. Skokan, The number of hypergraphs without linear cycles. J. Combin. Theory Ser. B 134 (2019), 309-321.

[5] J. Balogh and W. Samotij, The number of $K_{s, t}$-free graphs. J. Lond. Math. Soc. (2) 83 (2011), no. $2,368-388$.

[6] F. Behrend, On sequences of numbers not divisible one by another. Journal of the London Mathematical Society 1.1 (1935), 42-44.

[7] D. Conlon, An extremal theorem in the hypercube. Electron. J. Combin. 17 (2010), R111.

[8] D. Conlon and W. T. Gowers, Combinatorial theorems in sparse random sets. Annals of Mathematics (2016), 367-454.

[9] B. DeMarco, A. Hamm, and J. Kahn, On the triangle space of a random graph. Journal of Combinatorics. 4. 10.4310/JOC.2013.v4.n2.a4 (2012).

[10] B. DeMarco and J. Kahn, Mantel's Theorem for random graphs. Random Structures \& Algorithms. 47. 10.1002/rsa.20535 (2014).

[11] P. Erdős, On the combinatorial problems which I would most like to see solved. Combinatorica, 1(1) (1981), 25-42.

[12] P. Erdös, P. Frankl, and V. Rödl, The asymptotic number of graphs not containing a fixed subgraph and a problem for hypergraphs having no exponent. Graphs and Combinatorics, 2 (1986), 113-121. 
[13] P. Erdős, C. Ko and R. Rado, Intersection theorems for systems of finite sets. Quarterly Journal of Mathematics. Oxford. Second Series, 12 (1961), 313-320.

[14] F. Foucaud, M. Krivelevich, and G. Perarnau, Large subgraphs without short cycles. SIAM Journal on Discrete Mathematics 29 (2015), 65-78.

[15] P. Frankl and Z. Füredi, Exact solution of some Turán-type problems. Journal of Combinatorial Theory, Series A 45.2 (1987), 226-262.

[16] E. Friedgut, V. Rödl, and M. Schacht, Ramsey properties of random discrete structures. Random Structures \& Algorithms, 37(4) (2010), 407-436.

[17] Z. Füredi and M. Simonovits, The history of degenerate (bipartite) extremal graph problems. Erdős Centennial. Springer, Berlin, Heidelberg, (2013), 169-264.

[18] S. Glock, D. Kühn, A. Lo, and D. Osthus, The existence of designs via iterative absorption. arXiv preprint arXiv:1611.06827 (2016) .

[19] P. Keevash, The existence of designs. arXiv preprint arXiv:1401.3665 (2014).

[20] Y. Kohayakawa, B. Kreuter, and A. Steger, An extremal problem for random graphs and the number of graphs with large even-girth. Combinatorica 18 (1998), no. 1, 101-120.

[21] Y. Kohayakawa, T. Łuczak, and V. Rödl, On $K_{4}$-free subgraphs of random graphs. Combinatorica 17 (1997), no. 2, 173-213.

[22] W. Mantel, Problem 28 (Solution by H. Gouwentak, W. Mantel, J. Teixeira de Mattes, F. Schuh and W. A. Wythoff). Wiskundige Opgaven (1907), 10, 60-61.

[23] R. Morris and D. Saxton, The number of $C_{2 l}$-free graphs. Adv. Math. 298 (2016), 534-580.

[24] K. Roth, On certain sets of integers. J. London Math Soc. 28 (1953) , 104-109.

[25] V. Rödl and L. Thoma, Asymptotic packing and the random greedy algorithm. Random Structures \& Algorithms, 8(3) (1996), 161-177.

[26] I.Z. Ruzsa and E. Szemerédi, Triple systems with no six points carrying three triangles. Combinatorics (Keszthely, 1976), Coll. Math. Soc. J. Bolyai, 18 (1978), 939-945.

[27] D. Saxton and A. Thomason, Hypergraph containers. Inventiones mathematicae 201.3 (2015), 925-992.

[28] M. Schacht, Extremal results for random discrete structures. Annals of Mathematics 184(2) (2016), 333-365.

[29] P. Turán, On an extremal problem in graph theory. Matematikai és Fizikai Lapok, 48 (1941), $436-452$. 